University of Wollongong

Research Online

Faculty of Engineering and Information

Faculty of Engineering and Information

Sciences - Papers: Part A

Sciences

$1-1-2015$

\title{
An efficient background modeling approach based on vehicle detection
}

Jia-Yan Wang

Tianjin Polytechnic University

Limei Song

Tianjin Polytechnic University, lilymay1976@126.com

Jiangtao Xi

University of Wollongong, jiangtao@uow.edu.au

Qinghua Guo

Tianjin Polytechnic University, qguo@uow.edu.au

Follow this and additional works at: https://ro.uow.edu.au/eispapers

Part of the Engineering Commons, and the Science and Technology Studies Commons

Research Online is the open access institutional repository for the University of Wollongong. For further information contact the UOW Library: research-pubs@uow.edu.au 


\title{
An efficient background modeling approach based on vehicle detection
}

\begin{abstract}
The existing Gaussian Mixture Model(GMM) which is widely used in vehicle detection suffers inefficiency in detecting foreground image during the model phase, because it needs quite a long time to blend the shadows in the background. In order to overcome this problem, an improved method is proposed in this paper. First of all, each frame is divided into several areas(A, B, C and D), Where area A, B, C and D are decided by the frequency and the scale of the vehicle access. For each area, different new learning rate including weight, mean and variance is applied to accelerate the elimination of shadows. At the same time, the measure of adaptive change for Gaussian distribution is taken to decrease the total number of distributions and save memory space effectively. With this method, different threshold value and different number of Gaussian distribution are adopted for different areas. The results show that the speed of learning and the accuracy of the model using our proposed algorithm surpass the traditional GMM. Probably to the 50th frame, interference with the vehicle has been eliminated basically, and the model number only $35 \%$ to $43 \%$ of the standard, the processing speed for every frame approximately has a $20 \%$ increase than the standard. The proposed algorithm has good performance in terms of elimination of shadow and processing speed for vehicle detection, it can promote the development of intelligent transportation, which is very meaningful to the other Background modeling methods. (2015) COPYRIGHT Society of Photo-Optical Instrumentation Engineers (SPIE). Downloading of the abstract is permitted for personal use only.
\end{abstract}

Keywords

modeling, background, approach, efficient, vehicle, detection

Disciplines

Engineering | Science and Technology Studies

Publication Details

J. Wang, L. Song, J. Xi \& Q. Guo, "An efficient background modeling approach based on vehicle detection," in Proc. SPIE 9675, AOPC 2015: Image Processing and Analysis, 2015, pp. 96751A-1-96751A-6.

This conference paper is available at Research Online: https://ro.uow.edu.au/eispapers/4725 


\title{
An Efficient Background Modeling Approach Based on Vehicle
}

\section{Detection}

\author{
Jia-yan Wang ${ }^{1}$,Li-mei Song ${ }^{1}$,Jiang-tao Xi ${ }^{2}$,Qing-hua Guo ${ }^{1}$ \\ 1 Key Laboratory of Advanced Electrical Engineering and Energy Technology, Tianjin Polytechnic \\ University, Tianjin 300387, China \\ 2 School of Electrical, Computer and Telecommunications Engineering, University of Wollongong, \\ Keiraville 2500, Australia
}

\begin{abstract}
The existing Gaussian Mixture $\operatorname{Model}(\mathrm{GMM})$ which is widely used in vehicle detection suffers inefficiency in detecting foreground image during the model phase, because it needs quite a long time to blend the shadows in the background. In order to overcome this problem, an improved method is proposed in this paper. First of all, each frame is divided into several areas(A, B, C and D), Where area A, B, C and D are decided by the frequency and the scale of the vehicle access. For each area, different new learning rate including weight, mean and variance is applied to accelerate the elimination of shadows. At the same time, the measure of adaptive change for Gaussian distribution is taken to decrease the total number of distributions and save memory space effectively. With this method, different threshold value and different number of Gaussian distribution are adopted for different areas. The results show that the speed of learning and the accuracy of the model using our proposed algorithm surpass the traditional GMM. Probably to the 50th frame, interference with the vehicle has been eliminated basically, and the model number only $35 \%$ to $43 \%$ of the standard, the processing speed for every frame approximately has a $20 \%$ increase than the standard. The proposed algorithm has good performance in terms of elimination of shadow and processing speed for vehicle detection, it can promote the development of intelligent transportation, which is very meaningful to the other Background modeling methods.
\end{abstract}

Keywords: Background modeling, Vehicle detection, Gaussian Mixture Model(GMM)

\section{INTRODUCTION}

At present, benefited from the rapid development of computer vision, artificial intelligence technology and hardware technology, image and video technology has been widely used in the new generation of intelligent transportation system. Commonly algorithm of vehicle detection based on video are optical flow method, frame difference method and background subtraction method ${ }^{[12]}$. Background elimination method is one of the most important methods in vehicle detection ${ }^{[13}$. Common method has the mean background subtraction method, Gaussian average method, median method, kalman filter model law and Gaussian mixture model (GMM) method ${ }^{[4]}$. In view of the GMM has good practical effect, and wide application, this paper will focus on the algorithm and its improvement.

In recent years, a lot of research work has been done and make a number of achievements in research of the GMM. In Reference [5], one of the most commonly used approaches for updating GMM is presented by Stauffer and Grimson, however, when the moving object resting for a long time, which still appears invalid status. In Reference [6], Lu 
Qing-Hua built an adaptive learning rate schedule for each Gaussian distribution, calculate the ratio of each pixel adaptive learning algorithm to improve the convergence rate, but it needs the approximation for parameters update rate, which is not optimal algorithms. In Reference [7], Shimada reduce the number of Gaussian distribution by combining similar Gaussian distribution, although the amount of calculation is reduced, but processing accuracy has dropped a lot. There are still many other techniques using high level processing to assist the background modelling have been proposed, such as Ref[8] [9]etc. Based on the research of our laboratory[10-11], we also get a lot of inspiration. An improved algorithm is proposed based on the recent results. Not only the parameters including weight, mean and variance but also the number of the Gaussian distribution is adapted for each pixel constantly. By selecting the number of components for each pixel in an online process, the algorithm has good adaptability to the scene.

\section{THE PRINCIPLE OF GAUSSIAN MIXTURE MODEL}

In this section, we will analyze the principle of Stauffer and Grimson ${ }^{[5]}$ and its disadvantage. The authors model each background pixel with K Gaussian distributions (usually, K ranges from 3 to 5). The probability of the random variable $X_{t}$ at time $T$ can be expressed as

$$
P\left(X_{t}\right)=\sum_{K=1}^{K} \omega_{k, t} \eta_{k}\left(X_{t}, \mu_{t}, \sum_{k}\right)
$$

Where $\omega_{k, t}$ represents the weight parameter of the $\mathrm{k}^{\text {th }}$ Gaussian component, $\eta_{k}\left(X_{t}, \mu_{t}, \sum_{k}\right)$ represents the probability of the normal distribution of $\mathrm{k}^{\text {th }}$ Gaussian component can be expressed as

$$
\eta_{k}\left(X_{t}, \mu_{t}, \sum_{k}\right)=\frac{1}{(2 \pi)^{\frac{n}{2}}\left|\sum_{k}\right|^{\frac{1}{2}}} e^{-\frac{1}{2}\left(X_{t}-\mu_{t}\right)^{T} \sum_{k}^{-1}\left(X-\mu_{t}\right)}
$$

Where $\mu_{t}$ represents the mean value and $\sum_{k}=\sigma_{k}{ }^{2} I$ represents the covariance. It is time to judge which Gaussian distribution is most probable to blend in background model after model update. Based on the appropriate value $W_{k, t} / \sigma_{k}$ we can sort the $\mathrm{K}$ distributions and the first $\mathrm{B}$ distributions are chosen as a background model. $\mathrm{B}$ can be expressed as:

$$
B=\arg \min _{b}\left\{\sum_{K=1}^{b} \omega_{k, t}>T\right\}
$$

Where $\mathrm{T}$ is the threshold of the background model, which represents the minimum prior probability of the background. Every new pixel value is checked against the existing K Gaussian distributions until a match is found. The Gaussian component which has been identified as suitable will be updated and is represented by the following formula:

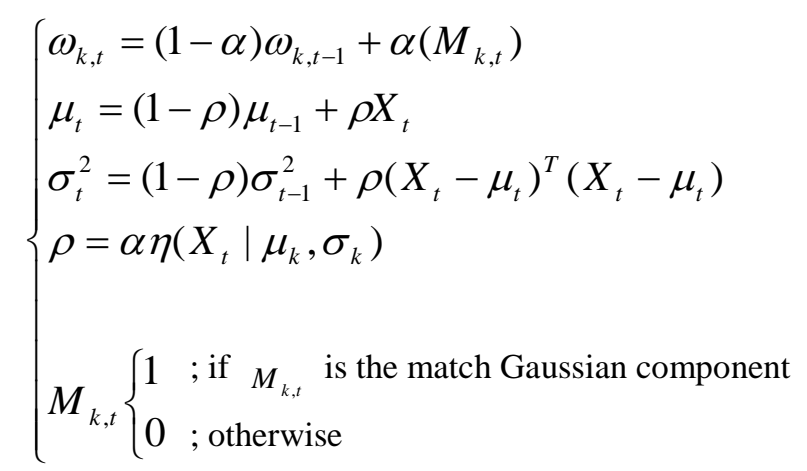

where $\alpha$ is the learning rate for weight and $M_{k, t}$ is 1 if the model which matched, otherwise it is $0, \rho$ is the learning rate for mean value and variance. 


\section{THE REVISED GAUSSIAN MIXTURE MODEL}

\subsection{Accelerate the elimination of shadows}

In actual traffic scene, there is often a movement of the vehicle in the 1st frame, After the initialization, the movement of the vehicles will be as a background, so when dealing with subsequent frames, the moving vehicle area for a long time as a prospect to extract, produce the so-called shadow. The existence of the shadow will affect the modeling background, impact on subsequent vehicle tracking and counting. The conventional GMM need quite a long time to blend the shadows in the background.

A method to accelerate the elimination of shadows is proposed in this paper, first of all, we divide each frame into several areas, as shown in figure 1 . Where area B, C and D are the places where vehicle through, and decided by the frequency and the scale of the vehicle access, area $\mathrm{A}$ is the external environment. And we also set up an initialization frames $\mathrm{T}$, on $\mathrm{T}$ frames adopt different parameters before and after the update method.

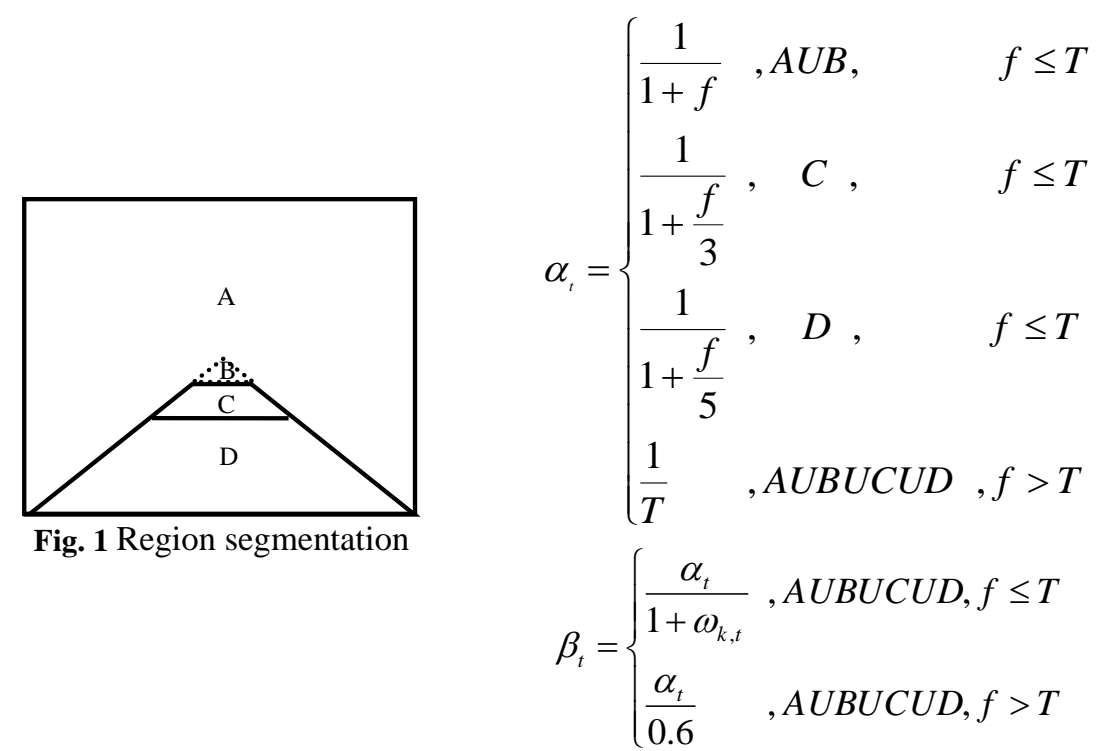

where $\alpha_{t}$ is the learning rate for weight, $\rho_{t}$ is the learning rate for mean and variance, $\mathrm{f}$ is the current video frames, AUB represents collection of regional A and B. We can see that, before the $\mathrm{T}$ frames, the weight and variance are bigger, so, Background are updated faster, can accelerate the elimination of shadows, and more important, it is found that the place where vehicles appear frequently and most influential is just the place where the detection is strong. After the $\mathrm{T}$ frames, learning rate for weights and variance is set to a constant, this will ensure that does not reduce the ability to adapt to environmental change, speed up the computation. According to experience, we make the value of $\mathrm{T}$ is 200 .

\subsection{Save memory space and reduce the execution time}

GMM will set up K Gaussian models for each pixel in a general way, actually this will cause the waste of memory space. Because the possibility of moving object is very small in many regions, often only need a single or a small amount of Gaussian distribution. Therefore, this paper proposes an improved method, on the basis of the Gaussian mixture model, for each pixel dynamic increase or decrease the number of Gaussian distribution but place a cap on each pixel. Initializing each pixel has only one Gaussian distribution, when the arrival of a new frame, if a match is not found in the distribution of the pixel, and the distribution of pixels is less than the upper limit value $\mathrm{M}$, then add a distribution to the pixel with the current value as the mean, and give a small weight and large variance. If the distribution is equal to the number of pixels on the limit of $\mathrm{M}$, then replace with a new distribution of the final distribution(take the minimum of $\left.W_{k} / \sigma_{k}\right)$. Due to the area $\mathrm{A}$ and $\mathrm{B}$ are large and have little change, the distribution of these weights will become very small, and the area $\mathrm{C}$ and $\mathrm{D}$ are the place where we care about most, if we adopt the same threshold value, then due to the number of pixels distribution does not reach the upper limit in the area A and B, the distribution will not be replaced, so the algorithm clear the distribution of the weight which is very small every L frames, and even more important, we make use of statistical histogram dividing different thresholds to reduce unnecessary distribution, save memory space, 
improve the processing speed.

\section{MEASUREMENTS PROCESSING AND RESULTS}

This section demonstrates the performance of the Stauffer and Grimson model ${ }^{[5]}$ and our proposed algorithms on an image sequence. To analyze the performance of the algorithm we used three dynamic scenes with fixed number of components $M=3$. The sequences were manually segmented to generate the ground truth, in order to better observations, the sequence shown here are 640X480 images at $30 \mathrm{fps}$. The programming environment is Visual Studio 2012 edition with OpenCV 2.4.9. The hardware environment is Intel 3.2GHz, RAM 4G.Before the improvements, $\alpha=0.003, \rho=0.01$. After the improvements, $T=200, \mathrm{~L}=50$. Figure 2 shows the result of shadow elimination. Figure 3 shows the time they take for the algorithm and the number of models.Figure 4 is a bar chart that displays the comparison of model numbers between standard and improved GMM.Figure 5 is a line chart that expresses the comparison of model numbers between standard and improved GMM.

We can see something to be expected from the figure. In the aspect of shadow elimination(we can refer to figure 1), before the improvements, four cars before the picture is initialized to the background, we also can see that the cars in the foreground already overlap. In the subsequent frames, they also stay in the background, at the same time,the four cars still appear in the foreground cause that make our detection error, basically disappear until the 200th frame. After the improvements, probably to the 50th frame, interference with the vehicle is basically eliminated, effect is remarkable, and save time for vehicle detection significantly.

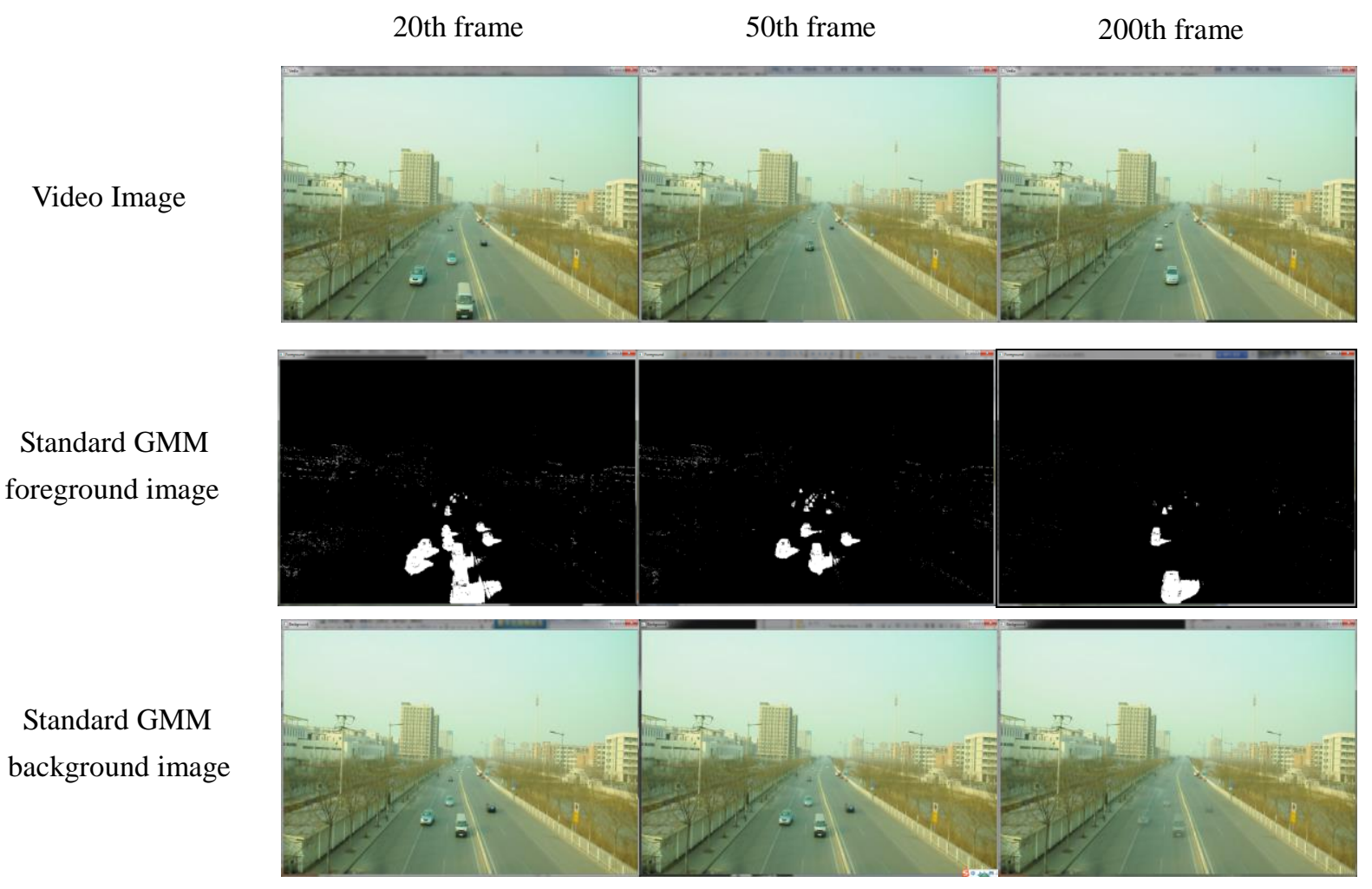


Improved GMM

foreground image

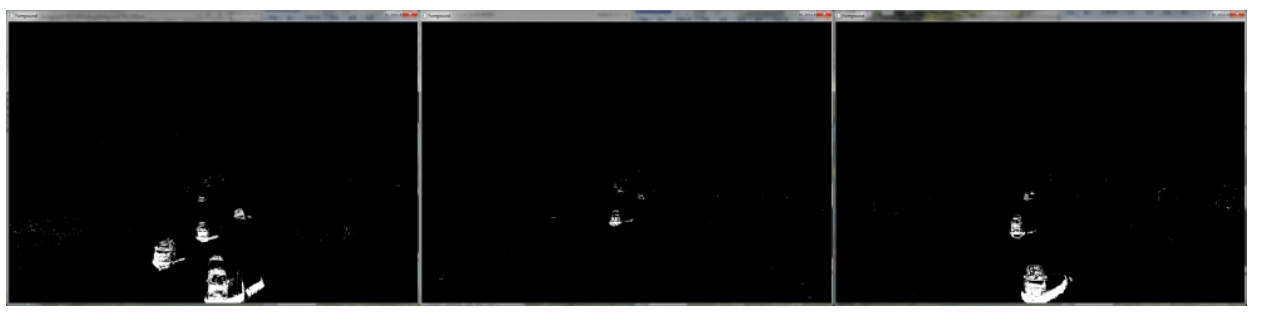

Improved GMM

background image

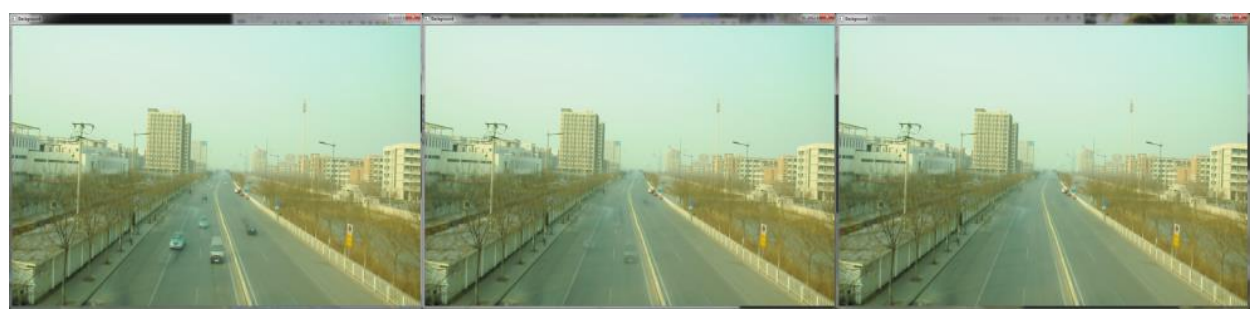

Fig. 2 The comparison of modeling between standard and improved GMM

\begin{tabular}{|c|c|c|c|c|c|c|}
\hline \multicolumn{2}{|c|}{ Video frame } & 20th frame & 50th frame & 100th frame & 150th frame & 200th frame \\
\hline \multirow{2}{*}{$\begin{array}{c}\text { computation } \\
\text { time/ms }\end{array}$} & Standard & 84 & 88 & 88 & 84 & 80 \\
\cline { 2 - 7 } & Improved & 65 & 66 & 67 & 68 & 67 \\
\hline \multirow{2}{*}{$\begin{array}{c}\text { Model } \\
\text { numbers }\end{array}$} & Standard & 921600 & 921600 & 921600 & 921600 & 921600 \\
\cline { 2 - 7 } & Improved & 324231 & 356810 & 361520 & 379243 & 395242 \\
\hline
\end{tabular}

Fig. 3 The comparison of computation time and model numbers

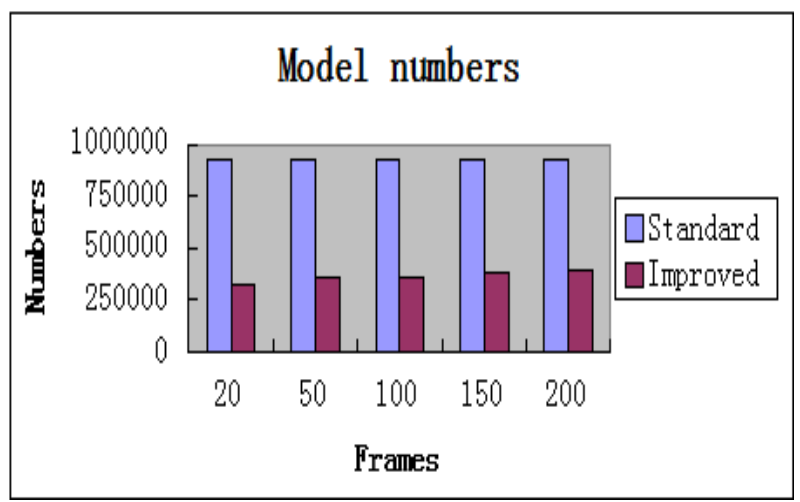

Fig. 4

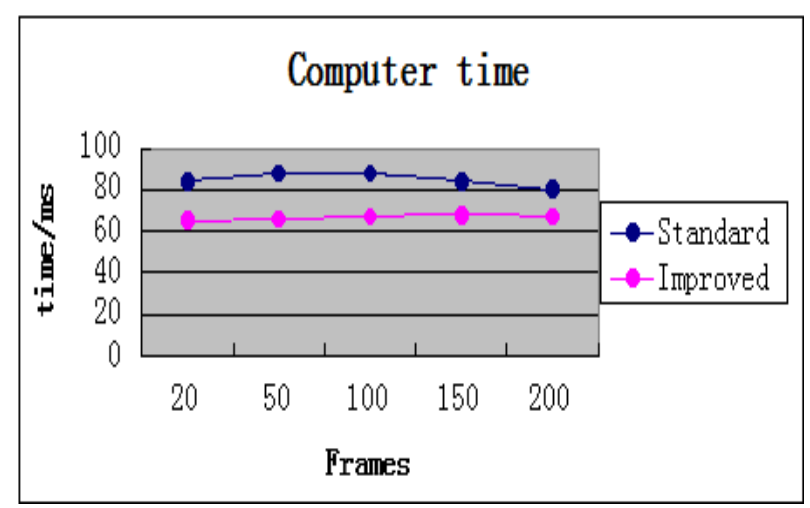

Fig. 5

Fig. 4 The comparison of model numbers between standard and improved GMM

Fig. 5 The comparison of computation time between standard and improved GMM

In the aspect of computation time and model numbers, it is concluded that the original data as shown in the figure 2. In order to observe conveniently, we make different figures for computation time and model numbers. Model numbers is shown in figure 3, we shall see a clean result that model number only $57 \%$ to $65 \%$ of the standard and save memory space effectively after the improvement. Computation time is shown in figure 4, the improved GMM significantly accelerate the processing speed base the trends of the computation time. All things considered, the improved GMM is doing pretty well as we have seen. 


\section{CONCLUSIONS}

We have presented new update algorithms for learning rate including weight, mean and variance to accelerate the elimination of shadows, at the same time,we take the measure of adaptive change for Gaussian distribution numbers to decrease the total number of distributions and save memory space effectively. The results show that the speed of learning and the accuracy of the model using our new update algorithm surpass the traditional GMM, it has good performance in terms of elimination of shadow and processing speed for vehicle detection.

\section{ACKNOWLEDGEMENT}

This research is supported by the National Natural Science Foundation of China (No. 60808020 and 61078041), the National Science and Technology Support (No.2014BAH03F01), the Tianjin Research Program of Application Foundation and Advanced Technology (No. 10JCYBJC07200), and the Technology program of Tianjin Municipal Education Commission (20130324). Thanks for the support from National Natural Science Foundation Committee and Tianjin Research Program of Application Foundation and Advanced Technology. This research is also supported by the Tianjin Key Laboratory of Advanced Electrical Engineering and Energy Technology.

\section{REFERENCE}

[1] Dong Chun-li, Dong Yu-ning, "Survey on video based vehicle detection and tracking algorithms," Journal of Nanjing University of Posts and Telecommunication(Natural Science), 29(2), 88-94(2009).

[2] Lin Chun-li, Wang Ke-jun, Xia Yu,et al, "Detection method of moving object with small displacement," Journal of Optoelectronics Laser, 22(3), 418-421(2011).

[3] Qian Jing, "Research of video-based vehicle detection algorithms through background subtraction," Shanghai Jiaotong University, 2007.

[4] Gan Xin-sheng, Zhao Shu-bin, "Comparison on background subtraction algorithms for moving target detection," Command Control \& Simulation, 30(3), 45-50(2008).

[5] Stauffer C, Grimson W E L, "Learning patterns of activity using real-time tracking," IEEE Transactions on Pattern Analysis and Machine Intelligence, 22(8), 747-757(2000).

[6] Lu Qing-hua, Wu Zhi-wei, "An improved mobile vehicle detection method based on Gaussian mixture model," Journal of Optoelectronics Laser, 24(4), 751-757(2013).

[7] Shimada A, Arita D, Taniguchi R, "Dynamic control of adaptive mixture-of-gaussions background mode," Proceeding of the IEEE International Conference on Video and Signal Based Surveillance, 2006.

[8] Varadarajan Sriram,Wang Hong-Bin, "Fast convergence of regularised Region-based Mixture of Gaussians for dynamic background modelling," Computer Vision and Image Understanding, April 15, 2014.

[9] Evangelio, Ruben Heras, "Adaptively splitted GMM with feedback improvement for the task of background subtraction," IEEE Transactions on Information Forensics and Security, 9(5), 863-874(2014).

[10] Song Limei, Dong Xiaoxiao, Xi Jiangtao, Yu Yanguang and Yang Chaokui, "A new phase unwrapping algorithm based on Three Wavelength Phase Shift Profilometry method," Optics and Laser Technology 45, 1(2013).

[11] Song Limei, Chang Yulan, Li Zongyan, Wang Pengqiang, Xing Guangxin and Xi Jiangtao, "Application of global phase filtering method in Multi frequency measurement," Optics Express 22, 11(2014). 